\title{
Dimensions of the optic chiasm: quantitative ultrasound comparison between fetuses with anophthalmia/microphthalmia and normal fetuses
}

\author{
Li-Hong Wü, Qiao Zheng", Miao He, Li-He Zhang, Liu Du, Hong-Ning Xie \\ Department of Ultrasonic Medicine, First Affiliated Hospital of Sun Yat-sen University, Guangzhou, China
}

Contributions: (I) Conception and design: LH Wu, HN Xie; (II) Administrative support: None; (III) Provision of study materials or patients: None; (IV) Collection and assembly of data: LH Wu, Q Zheng, M He, LH Zhang, L Du; (V) Data analysis and interpretation: LH Wu, Q Zheng; (VI) Manuscript writing: All authors; (VII) Final approval of manuscript: All authors.

"These authors contributed equally to this work.

Correspondence to: Hong-Ning Xie. Department of Ultrasonic Medicine, First Affiliated Hospital of Sun Yat-sen University, 58 Zhongshan Er Road, Guangzhou 510080, China. Email: xiehn@mail.sysu.edu.cn.

Background: The precise pathogenesis of anophthalmia/microphthalmia remains unknown. Prenatal observation of the optic chiasm in fetuses with this malformation would assist in understanding the embryonic development of the condition. The present study aimed to establish the normal fetal size ranges of decussation of the optic chiasm, optic nerves, and optic tracts in the axial plane using two-dimensional transabdominal ultrasound throughout gestation and to compare these ranges to the corresponding values in fetuses with anophthalmia/microphthalmia

Methods: In total, 310 normal fetuses and 16 fetuses with anophthalmia/microphthalmia were included in this study. The widths of the decussation of the optic chiasm, optic nerves, and optic tracts of normal fetuses at 19-40 weeks' gestation were measured in the axial plane by two-dimensional transabdominal ultrasound. The same widths were retrospectively measured in the axial plane using three-dimensional ultrasound in fetuses with anophthalmia/microphthalmia and compared to the results from the normal fetuses.

Results: The decussation, optic nerves, and optic tracts of 310 normal fetuses were measured. The normal widths of the decussation of the optic chiasm, optic nerves, and optic tracts increased linearly with gestational age. The interobserver and intraobserver reproducibility was excellent for the decussation but relatively low for the optic nerves and optic tracts. The optic nerve width of fetuses with anophthalmia/microphthalmia was significantly smaller than that of normal fetuses $(\mathrm{P}<0.001)$, but the widths of the decussation $(\mathrm{P}=0.061)$ and optic tracts $(\mathrm{P}=0.053)$ were not significantly different between the two groups.

Conclusions: The normal ranges of the decussation of the optic chiasm, optic nerves, and optic tracts established in this study can provide a quantitative basis for prenatal evaluation of the optic pathway. Fetal anophthalmia/microphthalmia may be associated with optic nerve hypoplasia.

Keywords: Prenatal diagnosis; ultrasound; optic chiasm; anophthalmia; microphthalmia

Submitted Feb 06, 2021. Accepted for publication May 07, 2021.

doi: 10.21037/qims-21-151

View this article at: http://dx.doi.org/10.21037/qims-21-151 


\section{Introduction}

Anophthalmia and microphthalmia are rare congenital ocular abnormalities with approximately 3 and 14 cases per 100,000 population, respectively $(1,2)$. Microphthalmia is suspected if a globe has a total axial length that is at least two standard deviations below the mean for the fetus's age (3). Anophthalmia refers to the complete absence of the globe in the presence of ocular adnexa (eyelids, conjunctiva, and lacrimal apparatus) $(3,4)$. Some consider anophthalmia/ microphthalmia to be on a spectrum of abnormalities $(3,4)$ with a complex etiology related to the precise stage of embryological development affected by infection and/or gene mutation (5). The precise pathogenesis of anophthalmia/microphthalmia remains unknown. Prenatal observation of the optic chiasm (OC) in fetuses with this malformation would assist in understanding the embryonic development of the condition. However, few prenatal ultrasound studies have been conducted to evaluate the OC of fetuses with anophthalmia/microphthalmia.

The OC is an $\mathrm{X}$-shaped structure (decussation) lying over the body of the sphenoid bone, typically above the diaphragma sellae (6). The optic tracts (OTs) are posterior to the $\mathrm{OC}$, and the optic nerves (ONs) are anterior to it. An assessment of the fetal OC and the evaluation of its width may help in counseling parents when a septo-optic dysplasia is suspected. The fetal OC can be directly assessed by magnetic resonance imaging (MRI) (7). Some prenatal studies have also been carried out to evaluate the OC using transabdominal and transvaginal ultrasound with or without three-dimensional (3D) imaging (8-12). However, few studies have used two-dimensional (2D) transabdominal ultrasound to explore the normal dimensions of the OC, ONs, and OTs during pregnancy.

This study aims to establish the normal range of sizes of the decussation, ONs, and OTs in the axial plane using 2D transabdominal ultrasound throughout gestation and compare these three parameters between anophthalmic/ microphthalmic fetuses and normal fetuses to reveal the OC embryonic development of fetuses with anophthalmia/ microphthalmia.

\section{Methods}

The study was conducted at a tertiary prenatal diagnostic center in the Department of Ultrasound of the First Affiliated Hospital of Sun Yat-sen University in Guangzhou, Guangdong. The study was conducted following the
Declaration of Helsinki (as revised in 2013). The Institutional Review Board of the First Affiliated Hospital of Sun Yat-sen University approved the study. All patients gave informed consent to participate in this study. The control group for the normal range of the fetal OC included morphologically normal vertex fetuses in low-risk pregnancies undergoing routine ultrasound examinations at 19-40 weeks' gestation (from December 2019 to February 2020). Each fetus underwent a detailed sonographic examination, including biometry and anatomy assessments. Exclusion criteria for the control group included fetuses with congenital malformations, growth abnormalities (intrauterine growth restriction or macrosomia), maternal pregnancy complications, or poor visualization of the fetal brain (due to maternal subcutaneous fat or an unsuitable fetal position). Menstrual age was calculated based on the first day of the last normal menstrual period, defined as regular cyclic menses without antecedent contraceptive use, and was confirmed by either first- or early second-trimester sonography. The decussation, ON, and OT widths of the normal fetuses were prospectively measured in the axial plane using $2 \mathrm{D}$ transabdominal ultrasound, while the three parameters of the anophthalmia/microphthalmia fetuses were retrospectively measured offline with 4D ultrasound software.

For each subject in the normal group, the fetal brain was scanned by $2 \mathrm{D}$ transabdominal ultrasound as described by the International Society of Ultrasound in Obstetrics and Gynecology (ISUOG) guidelines (13). The scanning was performed by a single experienced sonographer using a Samsung WS80A (Samsung Medison, Seoul, Korea) ultrasound machine with a $1-8-\mathrm{MHz}$ transabdominal transducer. The fetal OC was visualized and measured following recently published recommended practices (12). First, the operator obtained an axial image of the fetal head in the biparietal-diameter plane. The probe was then moved in the caudal direction. Color Doppler imaging was activated to identify the circle of Willis. The OC is located between the two anterior cerebral arteries. After the color Doppler imaging was switched off, the probe was rotated toward the frontal part of the head by approximately $20^{\circ}-$ $40^{\circ}$ until at least one orbit was visible; both the anterior orbit and the cerebral peduncles should always be identified. Its distinctive shape identified the OC. The probe was rotated slightly in this plane to visualize the anterior ONs or the posterior OTs. Finally, the width of the decussation was measured at the middle of the $\mathrm{X}$ shape (Figure 1A). Both sides of a segment of the anterior ONs or the posterior OTs were measured within $1 \mathrm{~cm}$ from the middle of the $\mathrm{X}$ 

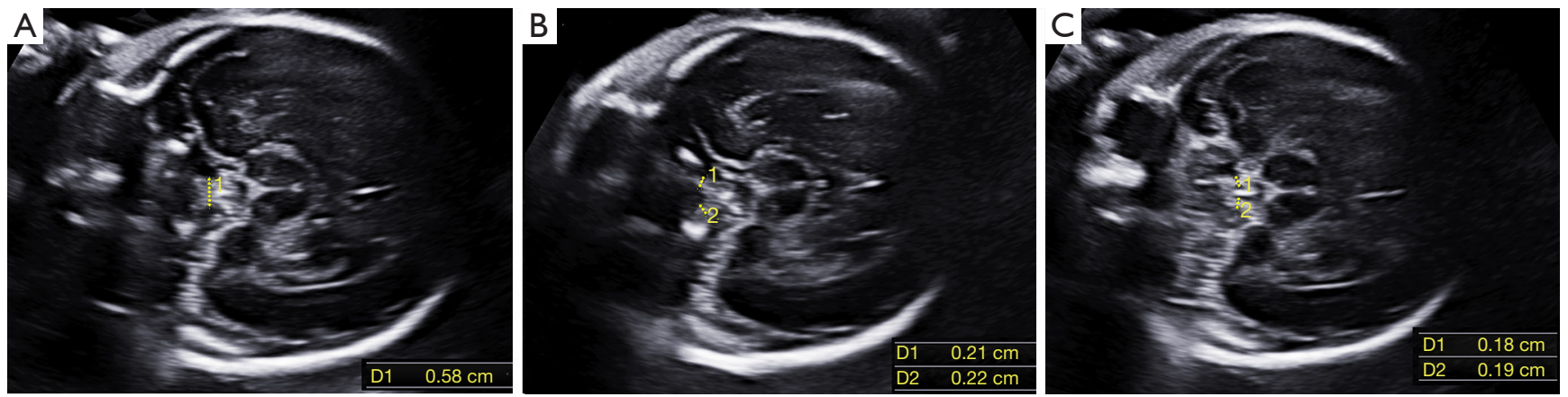

Figure 1 Two-dimensional transabdominal ultrasound image showing the measurement of a normal optic chiasm width in the axial plane for a $28^{+5}$-week fetus. (A) The measurement of the decussation width, (B) the measurement of the optic nerve width, (C) the measurement of the optic tract width.

shape (Figure 1B,C), and the two results were averaged. The measurements were repeated by a second sonographer using the cineloop saved on the ultrasound machine. The second sonographer was not aware of the first set of measurements.

The study group consisted of fetuses with suspected anophthalmia/microphthalmia at prenatal ultrasound scanning; the study group was recruited between January 2008 and December 2019. The diagnosis of anophthalmia/ microphthalmia was confirmed by autopsy. Fetuses with suspected anophthalmia/microphthalmia accompanied by other severe intracranial defects such as holoprosencephaly or encephalocele were excluded. The previously stored fetal brain volumes were retrospectively analyzed offline with 4D software (4D ViewTM, version 14 Ext.2, GE Medical Systems, Zipf, Austria) by an experienced sonographer (L-H W, >5 years' experience in fetal sonography). The acquisition of the fetal brain volumes and the offline analysis were performed with the GE Voluson Expert 730, E6, E8, and E10 (GE Medical Systems, Zipf, Austria) as follows: An acquisition box was placed over the biparietal diameter plane. A volume was obtained using the surface program set on "maximum quality", and a sweep was then performed in the sagittal plane with an angle of $30^{\circ}-45^{\circ}$, depending on the size of the fetus's head. The volumes were stored for offline analysis. The volumes were processed with dedicated software. Subsequently, the volume datasets were adjusted to optimize the tissue-contrast resolution. Cerebral peduncles can be visualized by scrolling from top to bottom, and the image was rotated toward the frontal part of the fetal head by approximately $20^{\circ}-40^{\circ}$ so that at least one orbit could be seen. The OC was then identified by its shape (Figure 2). Measurements of the decussation, $\mathrm{ONs}$, and OTs were treated as described in the 2D case.
Agreement between the normal measurements of the two sonographers using $2 \mathrm{D}$ transabdominal ultrasound was analyzed using the intraclass correlation coefficient (ICC). Intraobserver variation was calculated from 30 cases, with the difference in gestational age (GA) between measurements being 1 week. The correlation between the GA and the decussation of the OC, ON, and OT measurements in the normal group was determined through linear regression analysis. Nomograms were created for the three parameters to display the differences in distribution between the two groups. The three parameters were compared between the normal and anophthalmia/ microphthalmia fetuses by multiple linear regression. All analyses were conducted with SPSS software 16.0 (SPSS Inc., Chicago, IL, USA). The statistical significance was defined as $\mathrm{P}<0.05$.

\section{Results}

A total of 361 normal fetuses met the inclusion criteria for this study. However, 44 normal fetal ONs were unable to be clearly displayed. Of these, 26 fetal ONs that we failed to measure successfully had shadowing from the sphenoid bone (median GA of $35^{+6}$, range: $33^{+1}$ to $39^{+6}$ ). The GA of a further 18 fetuses was too early to distinguish the $\mathrm{ON}$, or the fetuses were in an unsuitable position (angle to occiput anterior). Finally, the OC decussations and the OTs and ONs of 310 normal fetuses were successfully measured to establish the normal range. The fetuses were scanned at a median GA of $30^{+2}$ weeks (range: $19^{+4}$ to $40^{+1}$ weeks). Interobserver and intraobserver reliability were evaluated according to the ICCs of the measurements. As shown in Table 1, the ICCs between the two observers were 

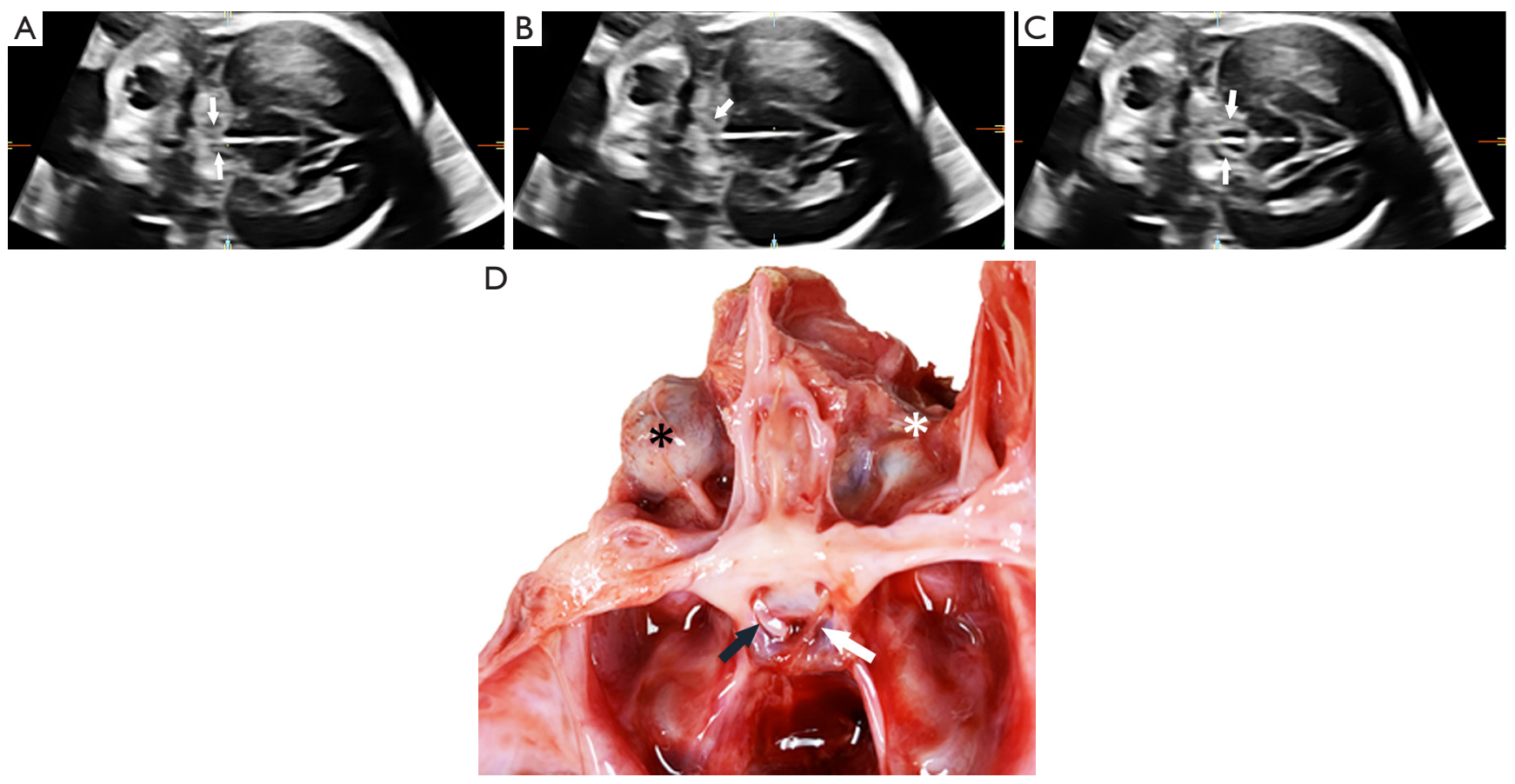

Figure 2 Three-dimensional ultrasound image and pathology specimens showing the optic chiasm in the axial plane for a $22^{+2}$-week fetus with unilateral microphthalmia (Case 4). (A) Sonograms showing a normal-shaped decussation (arrows). (B) Sonograms showing that the left side optic nerve (arrow) is visible, but the right side optic nerve is invisible. (C) Sonograms showing bilateral normal-shaped optic tracts (arrows). (D) Pathology specimens showing the right microphthalmia (white*) and ipsilateral delicate optic nerve (white arrow), and the left normal-shaped eye (black *) and optic nerve (black arrow).

Table 1 Interobserver and intraobserver reliability of the decussation, ON, and OT measurements

\begin{tabular}{lcc}
\hline Parameter & Interobserver ICC $(95 \% \mathrm{Cl})$ & Intraobserver ICC $(95 \% \mathrm{Cl})$ \\
\hline Decussation & $0.901(0.878-0.920)$ & $0.885(0.858-0.907)$ \\
ON & $0.798(0.623-0.897)$ & $0.775(0.526-0.893)$ \\
OT & $0.875(0.843-0.900)$ & $0.780(0.558-0.889)$ \\
\hline
\end{tabular}

$\mathrm{Cl}$, confidence interval; ICC, intraclass correlation coefficient; ON, optic nerve; OT, optic tract.

$0.901,0.798$, and 0.875 for the decussation, $\mathrm{ON}$, and OT measurements, respectively. The corresponding intraobserver ICCs were $0.885,0.775$, and 0.780 .

The $3 \mathrm{rd}, 5 \mathrm{th}, 50 \mathrm{th}, 95 \mathrm{th}$, and $97 \mathrm{th}$ centiles for the normal fetal decussation, ON, and OT measurements at 19-40 weeks in our cohort of fetuses are shown in Tables 2-4, respectively. The normal decussation, ONs, and OTs were strongly correlated with GA and increased linearly over time. The linear regression models are presented in Table 5.

Figure 3 displays the inclusion flowchart for the anophthalmia/microphthalmia fetuses. Anophthalmia/ microphthalmia was suspected in 27 fetuses based on the prenatal ultrasound. Four cases were lost to followup. The remaining 23 cases opted for termination of pregnancy. Anophthalmia/microphthalmia was confirmed at autopsy, of which seven cases were excluded: three due to holoprosencephaly, one due to frontal encephalocele, and three due to the inability to acquire good-quality $3 \mathrm{D}$ volume datasets to assess the OC. Our final study population comprised 16 fetuses with anophthalmia/ microphthalmia (Table 6).

Further, of the 16 abnormal fetuses, two had anophthalmia associated with microphthalmia, two had cerebral ventriculomegaly, one had agenesis of the corpus 
Table 2 Normal reference range for the decussation of the optic chiasm width at 19-40 weeks' gestation

\begin{tabular}{|c|c|c|c|c|c|c|}
\hline GA (week) & $\mathrm{n}$ & \multicolumn{5}{|c|}{ Decussation width (mm) } \\
\hline $19-21$ & 46 & 2.94 & 3.04 & 3.95 & 4.83 & 4.90 \\
\hline $22-24$ & 26 & 4.30 & 4.30 & 4.90 & 5.43 & 5.50 \\
\hline $25-27$ & 36 & 5.02 & 5.17 & 5.70 & 6.32 & 6.39 \\
\hline $31-33$ & 48 & 6.05 & 6.19 & 6.90 & 7.20 & 7.25 \\
\hline $34-36$ & 65 & 6.80 & 7.07 & 7.80 & 8.36 & 8.80 \\
\hline $37-40$ & 25 & 7.30 & 7.35 & 8.05 & 8.67 & 8.70 \\
\hline
\end{tabular}

GA, gestational age.

Table 3 Normal reference range for the fetal optic nerve width at 19-40 weeks' gestation

\begin{tabular}{|c|c|c|c|c|c|c|}
\hline GA (week) & $\mathrm{n}$ & \multicolumn{5}{|c|}{ ON width $(\mathrm{mm})$} \\
\hline $19-21$ & 46 & 1.20 & 1.20 & 1.60 & 1.87 & 1.90 \\
\hline $22-24$ & 26 & 1.30 & 1.34 & 1.75 & 2.10 & 2.10 \\
\hline $25-27$ & 36 & 1.70 & 1.70 & 2.10 & 2.60 & 2.60 \\
\hline $31-33$ & 48 & 2.10 & 2.10 & 2.60 & 3.16 & 3.20 \\
\hline $34-36$ & 65 & 2.70 & 2.73 & 3.20 & 3.76 & 4.00 \\
\hline $37-40$ & 25 & 3.10 & 3.13 & 3.40 & 4.20 & 4.30 \\
\hline
\end{tabular}

GA, gestational age; ON, optic nerve.

Table 4 Normal reference range for the fetal optic tract width at $19-40$ weeks' gestation

\begin{tabular}{|c|c|c|c|c|c|c|}
\hline GA (week) & $\mathrm{n}$ & \multicolumn{5}{|c|}{ OT width (mm) } \\
\hline $19-21$ & 46 & 1.12 & 1.20 & 1.65 & 2.31 & 2.46 \\
\hline $22-24$ & 26 & 1.40 & 1.41 & 1.80 & 2.28 & 2.30 \\
\hline $25-27$ & 36 & 1.70 & 1.70 & 2.00 & 2.53 & 2.60 \\
\hline $31-33$ & 48 & 2.10 & 2.13 & 2.50 & 2.90 & 2.97 \\
\hline $34-36$ & 65 & 2.75 & 2.80 & 3.15 & 3.91 & 4.00 \\
\hline $37-40$ & 25 & 3.10 & 3.11 & 3.40 & 3.80 & 3.80 \\
\hline
\end{tabular}

GA, gestational age; OT, optic tract. 
Table 5 Summary of the regression analysis of the decussation, optic nerve (ON), and optic tract (OT) measurements against gestational age (GA)

\begin{tabular}{lccc}
\hline Parameter & Linear regression models & $R^{2}$ & $r(P)$ \\
\hline Decussation & $-0.626+(0.238 \times \mathrm{GA})$ & 0.927 & $0.963(<0.001)$ \\
ON & $-0.471+(0.101 \times \mathrm{GA})$ & 0.813 & $0.901(<0.001)$ \\
OT & $-0.775+(0.111 \times \mathrm{GA})$ & 0.860 & $0.927(<0.001)$ \\
\hline
\end{tabular}

GA, gestational age; ON, optic nerve; OT, optic tract.

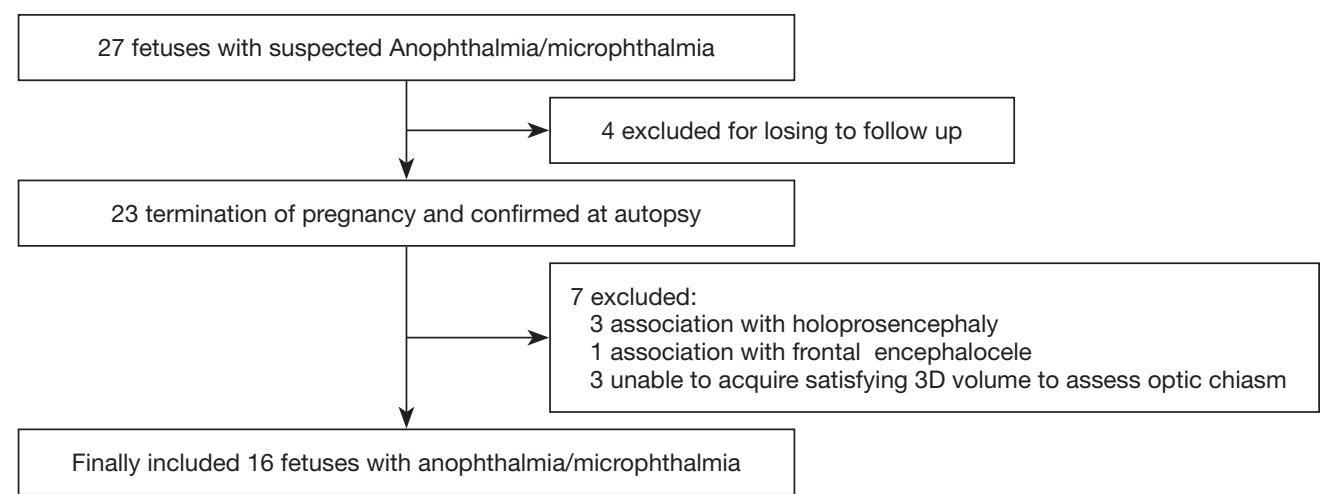

Figure 3 The flowchart of inclusion criteria for fetuses with anophthalmia/microphthalmia.

Table 6 Summary of the measurements of the decussation, ON, and OT in 16 fetuses with anophthalmia/microphthalmia

\begin{tabular}{|c|c|c|c|c|c|}
\hline Case & GA (week) & Ultrasound diagnosis & $\begin{array}{l}\text { Decussation } \\
\text { width }(\mathrm{mm})\end{array}$ & $\begin{array}{c}\text { ON width }(\mathrm{mm}), \\
\text { right/left }\end{array}$ & $\begin{array}{c}\text { OT width }(\mathrm{mm}), \\
\text { right/left }\end{array}$ \\
\hline 1 & $18^{+2}$ & Right microphthalmia; ACC; polycystic kidney disease & 2.5 & $0.7 / 1.1$ & $1.1 / 1.2$ \\
\hline 3 & $23^{+2}$ & Right microphthalmia; left anophthalmia & 4.3 & $0 / 0$ & $1.7 / 1.8$ \\
\hline 4 & $22^{+2}$ & Right microphthalmia & 4.3 & $0 / 1.7$ & $1.5 / 1.5$ \\
\hline 6 & $23^{+3}$ & Right microphthalmia & 4.6 & $1.5 / 1.7$ & $1.8 / 1.7$ \\
\hline 7 & $23^{+3}$ & Left microphthalmia & 5.2 & $1.9 / 1.0$ & $2.1 / 2.2$ \\
\hline 8 & $24^{+6}$ & Right microphthalmia; VSD & 5.2 & $1.2 / 1.5$ & $1.8 / 1.8$ \\
\hline 12 & 28 & Left microphthalmia; bilateral ventriculomegaly; CoA & 6.3 & $2.4 / 2.3$ & $2.4 / 2.5$ \\
\hline 13 & $31^{+2}$ & Right microphthalmia; left ectrodactyly; right single umbilical artery & 6.7 & $0 / 2.6$ & $2.7 / 2.8$ \\
\hline 14 & $31^{+5}$ & Right microphthalmia & 6.9 & $1.5 / 2.4$ & $2.4 / 2.4$ \\
\hline 15 & $33^{+6}$ & Right microphthalmia & 7.1 & $1.7 / 3.0$ & $3.3 / 2.7$ \\
\hline 16 & $34^{+3}$ & Right microphthalmia; PLSVC & 7.3 & $2.2 / 3.2$ & $3.0 / 3.0$ \\
\hline
\end{tabular}

ACC, agenesis of the corpus callosum; CoA, coarctation of the aorta; GA, gestational age; ON, optic nerve; OT, optic tract; PLSVC, persistent left superior vena cava; VSD, ventricular septal defect. 

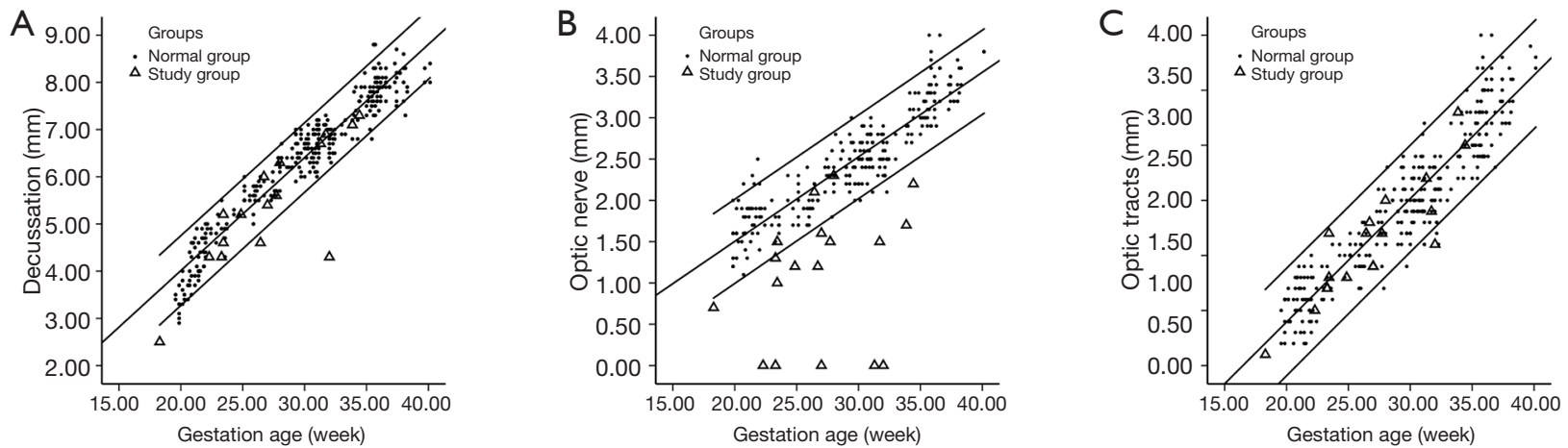

Figure 4 Plots showing the distribution of (A) the decussation, (B) optic nerve, and (C) optic tract in fetuses with anophthalmia/ microphthalmia $(\triangle)$ and normal fetuses $(\bullet)$. In $(A-C)$, horizontal lines show the normal mean and $95 \%$ range.

Table 7 Summary of the multiple linear regression of the decussation, optic nerve, and optic tract measurements between the normal and anophthalmia/microphthalmia fetuses

\begin{tabular}{|c|c|c|c|c|c|}
\hline Parameter & Independent variable & $\beta$ & $\mathrm{t}$ & $95 \% \mathrm{Cl}$ & $P$ \\
\hline \multirow{2}{*}{ Decussation } & GA & 0.239 & 59.777 & $(0.232--0.247)$ & $<0.001$ \\
\hline & Group & -0.337 & -3.292 & $(-0.539--0.136)$ & 0.061 \\
\hline \multirow[t]{2}{*}{ ON } & (constant) & 0.680 & 4.750 & & $<0.001$ \\
\hline & Group & -1.175 & -14.998 & $(-1.392--1.021)$ & $<0.001$ \\
\hline \multirow[t]{3}{*}{ OT } & (constant) & -0.676 & -5.702 & & $<0.001$ \\
\hline & GA & 0.111 & 38.830 & $(0.105--0.116)$ & $<0.001$ \\
\hline & Group & -0.146 & -2.112 & $(-0.282--0.10)$ & 0.053 \\
\hline
\end{tabular}

$\mathrm{Cl}$, confidence interval; GA, gestational age; ON, optic nerve; OT, optic tract

callosum (ACC), and eight were diagnosed with an extracranial abnormality. Therefore, a total of 18 deformed eyes were included in the study. The median GA at examination was $26^{+6}$ weeks (range: $18^{+2}$ to $34^{+3}$ weeks).

The decussation, ON, and OT measurements in the abnormal and normal groups are plotted against GA in Figure 4. The ON measurements enable sufficient separation between the two groups in the nomogram. However, the decussation and OT measurements demonstrated no difference in distribution between the abnormal and normal groups. Furthermore, $72.2 \%$ (13 out of 18) of the ON measurements of the abnormal group fell outside the $95 \%$ normal range. Comparison results of the two groups are shown in Table 7 . The ON width was significantly correlated with gestational age $(\beta=0.100$, $\mathrm{P}<0.001)$ and group $(\beta=-1.175, \mathrm{P}<0.001)$. The $\mathrm{ON}$ width of the anophthalmia/microphthalmia group was smaller than that of the normal group. However, the decussation $(\beta=-0.337, \mathrm{P}=0.061)$ and $\mathrm{OT}(\beta=-0.146, \mathrm{P}=0.053)$ widths showed no statistical difference between the two groups.

\section{Discussion}

The $\mathrm{OC}$ is a commissure formed by the anteriorly converging ONs and posteriorly diverging OTs. The development of decussation begins between the fourth and sixth week of gestation and is later continuous with the ONs and part of the OTs (6). The ONs develop in the substance of the optic stalk, which becomes apparent at the fourth week of gestation (6). The ONs has four sections: the intraocular, intraorbital, intraosseous, and intracranial portions. The intracranial $\mathrm{ON}$ sections measured in this 
study do not lie in a horizontal plane; rather, they rise upward from the optic canals at an angle of $15^{\circ}-45^{\circ}(6)$. Therefore, visualizing the intracranial ONs requires rotating the transducer upward at the base to view the decussation plane. The OTs, which are fully formed by the 13th week of gestation, are part of the afferent visual pathway between the chiasm and lateral geniculate nucleus (6).

Recently, it has been reported that $3 \mathrm{D}(8,9)$ and $2 \mathrm{D}(10-12)$ imaging can be used to observe the decussation and OTs. A previous study has evaluated the decussation with $2 \mathrm{D}$ transvaginal-ultrasound imaging in a coronal view (10). An echoanatomic correlation study by Paladini et al. (11) has confirmed that the structure identified as the OC on ultrasound is indeed the anatomical structure. In this study, we adopted the methodology described by Alonso et al. (12), which assesses the decussation, intracranial ONs, and OTs in the axial plane by $2 \mathrm{D}$ transabdominal ultrasound. Our results demonstrated excellent intraobserver repeatability and interobserver reproducibility on the decussation measurements, which are more reproducible than those of the OTs and the ONs.

Previous studies have suggested that measurement of the ONs is not feasible because of shadowing from the sphenoid bone $(8,11)$. However, it was reported in a recent article (12) that the ONs could be visualized on transabdominal ultrasound. Our results showed an 85.8\% (310/361) success rate for measuring normal intracranial ONs, although the repeatability test result of fetal $\mathrm{ON}$ measurement was not satisfactory (interobserver ICC 0.798 , intraobserver ICC $0.775)$. The fetal ONs were unsuccessfully measured in only 44 normal fetuses, most of which had a GA greater than 33 weeks and were thus prone to shadowing by the sphenoid bone or frontal bone. Because ultrasonic technology and penetration have been enhanced in recent years, measurement of the fetal ONs is feasible with an appropriate fetal position, especially in the late second and early third trimesters. However, the intraobserver and interobserver reproducibility needs to be improved.

A size abnormality in the chiasm can indicate various disorders, the most common being septo-optic dysplasia. Establishing a normal reference range can provide a quantitative basis for prenatal assessment of the OC as a diagnostic tool. Bault et al. constructed a similar range of normal values for the OT by measuring 98 normal fetuses at 21-36 weeks' gestation using 3D ultrasound (9); however, processing the fetal brain volume dataset proved relatively intricate and, thus, inconvenient for routine clinical practice. Vinals et al. measured the decussation of 115 normal fetuses between 21-30 weeks' gestation on the coronal plane with $2 \mathrm{D}$ transvaginal ultrasound and documented the normal range of the decussation width (10). However, it is not easy to obtain sonograms in the coronal plane of the fetal brain in routine clinical settings, and the use of transvaginal ultrasound in routine fetal screening is not generally accepted. In this study, the widths of the decussation, ONs, and OTs were measured in the axial plane using $2 \mathrm{D}$ transabdominal ultrasound. This technique may be put into practice more easily by clinical sonographers.

Normal eye development involves a complicated interplay between different ocular tissues arising from the ectoderm, neural crest cells, and mesenchyme (14). Two small grooves develop in the neural folds on the sides of the developing forebrain in approximately the third embryological week. Extending from the forebrain toward the surface ectoderm, they eventually become optic vesicles. As they grow, they remain connected to the forebrain by structures known as optic stalks in the earlier stages of development and optic nerves in the later stages (14). Some scholars have assumed that an interruption of embryogenesis within the first few weeks of gestation is the main cause of congenital anophthalmia/microphthalmia, encompassing aplasia of the eyeballs and hypoplasia of the optic pathway (15). Observations of the anophthalmic/ microphthalmic fetal optic pathway may find evidence of a partially developed eye regressing rather than aplasia of the optic vesicle (3). MRI has revealed agenesis of the optic pathways in some pediatric and adult cases (15-17). However, to the best of our knowledge, no previous studies have evaluated the optic pathways of anophthalmic/ microphthalmic fetuses, as we have done in this study.

Our study of 16 such fetuses found that the majority had abnormally small ONs; however, the widths of the decussations and the OTs were not different from those of normal fetuses. Cases 3 and 9 in this study were diagnosed as microphthalmia-associated with anophthalmia, where the optic nerve failed to develop on the side of anophthalmia. However, the decussation and OT widths of the two cases were within the normal range. We assumed that the more severe the microphthalmia, the smaller the optic nerve width. We speculate that fetal anophthalmia/ microphthalmia frequently combines with $\mathrm{ON}$ hypoplasiathe failure of the anterior neural tube to develop may be the pathogenesis of congenital anophthalmia/microphthalmia. However, due to the small number of anophthalmia/ microphthalmia cases in this study, we will require more data to confirm these assumptions. 
An important limitation of this study is the relatively small number of abnormal cases evaluated-only 16 fetuses with anophthalmia/microphthalmia were identified. However, these are rare congenital malformations; hence, this limitation is hard to overcome. Another limitation is that the widths of the decussation, ONs, and OTs for healthy fetuses were measured by 2D sonography, but those of the 16 abnormal fetuses were retrospectively analyzed by $3 \mathrm{D}$ methods. However, 2D ultrasound is as reliable as $3 \mathrm{D}$ ultrasound for visualization of the OC (11). We are conducting a prospective study to verify the current findings.

To conclude, the normal range of measurements for the decussation, ONs, and OTs that we have established can provide a quantitative basis for prenatal evaluation of the optic pathway. Fetal anophthalmia/microphthalmia is frequently associated with ON hypoplasia, and this finding may assist in understanding the embryonic pathology of congenital anophthalmia/microphthalmia.

\section{Acknowledgments}

Funding: This work was supported by funding from the National Natural Science Foundation of China, No. 81701459 .

\section{Footnote}

Conflicts of Interest: All authors have completed the ICMJE uniform disclosure form (available at http://dx.doi. org/10.21037/qims-21-151). The authors have no conflicts of interest to declare.

Ethical Statement: The authors are accountable for all aspects of the work in ensuring that questions related to the accuracy or integrity of any part of the work are appropriately investigated and resolved. The study was conducted in accordance with the Declaration of Helsinki (as revised in 2013). The Institutional Review Board of the First Affiliated Hospital of Sun Yat-sen University approved the study. All patients gave informed consent to participate in this study.

Open Access Statement: This is an Open Access article distributed in accordance with the Creative Commons Attribution-NonCommercial-NoDerivs 4.0 International License (CC BY-NC-ND 4.0), which permits the noncommercial replication and distribution of the article with the strict proviso that no changes or edits are made and the original work is properly cited (including links to both the formal publication through the relevant DOI and the license). See: https://creativecommons.org/licenses/by-nc-nd/4.0/.

\section{References}

1. Campbell H, Holmes E, MacDonald S, Morrison D, Jones I. A capture-recapture model to estimate prevalence of children born in Scotland with developmental eye defects. J Cancer Epidemiol Prev 2002;7:21-8.

2. Morrison D, FitzPatrick D, Hanson I, Williamson K, van Heyningen V, Fleck B, Jones I. National study of microphthalmia, anophthalmia, and coloboma (MAC) in Scotland: investigation of genetic aetiology. J Med Genet 2002;39:16-22.

3. Verma AS, Fitzpatrick DR. Anophthalmia and microphthalmia. Orphanet J Rare Dis 2007;2:47.

4. Guercio JR, Martyn LJ. Congenital malformations of the eye and orbit. Otolaryngol Clin North Am 2007;40:113-40.

5. Searle A, Shetty P, Melov SJ, Alahakoon TI. Prenatal diagnosis and implications of microphthalmia and anophthalmia with a review of current ultrasound guidelines: two case reports. J Med Case Rep 2018;12:250.

6. Joseph F, Rizzo I. Embryology, Anatomy, and Physiology of the Afferent Visual Pathway. In: Miller N, Newman $\mathrm{N}$, ^editors. In Clinical Neuroophthalmology. Maryland: Lippincott Williams \& Wilkin 2005. p. 34-42.

7. Parravano JG, Toledo A, Kucharczyk W. Dimensions of the optic nerves, chiasm, and tracts: MR quantitative comparison between patients with optic atrophy and normals. J Comput Assist Tomogr 1993;17:688-90.

8. Bault JP. Visualization of the fetal optic chiasma using three-dimensional ultrasound imaging. Ultrasound Obstet Gynecol 2006;28:862-4.

9. Bault JP, Salomon LJ, Guibaud L, Achiron R. Role of three-dimensional ultrasound measurement of the optic tract in fetuses with agenesis of the septum pellucidum. Ultrasound Obstet Gynecol 2011;37:570-5.

10. Viñals F, Ruiz P, Correa F, Goncalves PP. Two-dimensional visualization and measurement of the fetal optic chiasm: improving counseling for antenatal diagnosis of agenesis of the septum pellucidum. Ultrasound Obstet Gynecol 2016;48:733-8.

11. Paladini D, Birnbaum R, Donarini G, Maffeo I, Fulcheri E. Assessment of fetal optic chiasm: an echoanatomic and reproducibility study. Ultrasound Obstet Gynecol 2016;48:727-32. 
12. Alonso I, Azumendi G, Romero M, Anderica JR, Herrero JR, Azumendi P, Borenstein, M. Fetal optic chiasm: three steps for visualization and measurement on routine transabdominal ultrasound. Ultrasound Obstet Gynecol 2019;54:135-6.

13. Salomon LJ, Alfirevic Z, Berghella V, Bilardo C, Hernandez-Andrade E, Johnsen SL, Kalache K. Practice guidelines for performance of the routine mid-trimester fetal ultrasound scan. Ultrasound Obstet Gynecol 2011;37:116-26.

14. Ort V, Howard D, Development of the Eye: NYU School of Medicine, 2017. Available online: http://education.med. nyu.edu/courses/macrostructure/lectures/lec_images/eye. html

15. Aktekin M, Oz O, Saygili MR, Kurtoglu Z. Bilateral congenital anophthalmos and agenesis of the optic pathways. Yonsei Medical Journal 2005;46:296-9.

16. Sanjari MS, Ghasemi FK, Parvaresh MM, Kharazi HH, Kashkooli MB. Bilateral aplasia of the optic nerve, chiasm, and tracts in an otherwise healthy infant. Br J Ophthalmol 2006;90:513-4.

17. Albernaz VS, Castillo M, Hudgins PA, Mukherji SK. Imaging findings in patients with clinical anophthalmos. AJNR Am J Neuroradiol 1997;18:555-61.
Cite this article as: Wu LH, Zheng Q, He M, Zhang LH, Du L, Xie HN. Dimensions of the optic chiasm: quantitative ultrasound comparison between fetuses with anophthalmia/ microphthalmia and normal fetuses. Quant Imaging Med Surg 2021;11(10):4389-4398. doi: 10.21037/qims-21-151 